\title{
COVID-19 in a kidney transplant recipient treated with eculizumab for atypical hemolytic uremic syndrome: a case report
}

\author{
Noëlle Cognard ${ }^{1}\left(\mathbb{D} \cdot\right.$ Gabriela Gautier-Vargas $^{1} \cdot$ Peggy Perrin $^{1} \cdot$ Ilies Benotmane $^{1} \cdot$ Sophie Caillard ${ }^{1}$
}

Received: 14 January 2021 / Accepted: 19 April 2021 / Published online: 17 May 2021

(c) Italian Society of Nephrology 2021

$\begin{array}{ll}\text { Abbreviations } \\ \text { aHUS } & \text { Atypical Hemolytic Uremic Syndrome } \\ \text { COVID-19 } & \text { Coronavirus Disease 2019 } \\ \text { CT } & \text { Computed Tomography } \\ \text { KT } & \text { Kidney Transplantation } \\ \text { MMF } & \text { Mycophenolate Mofetil } \\ \text { SARS-CoV-2 } & \text { Severe Acute Respiratory Syndrome } \\ & \text { Coronavirus-2 }\end{array}$

\section{The case}

This report regards a woman who was taken in charge since 2002 (when she was aged 46 years) for severe acute kidney failure. Kidney biopsy revealed thrombotic microangiopathy and intravascular hemolysis. She was diagnosed with atypical hemolytic uremic syndrome (aHUS) caused by a heterozygous mutation (Gly324Glu, exon 9) in the gene encoding complement factor I, located on chromosome $4 q 25$. Despite treatment with plasmapheresis and methylprednisolone, hemodialysis was performed in 2007 because of progressive renal function deterioration, and she underwent a kidney transplantation (KT) in 2014. Her immunosuppressive regimen consisted of thymoglobulin, tacrolimus, mycophenolate mofetil (MMF), and steroids. Eculizumab was prescribed to prevent aHUS recurrences. The initial maintenance dose was $1200 \mathrm{mg}$ every two weeks. Graft function was stable (serum creatinine: $130 \mu \mathrm{mol} / \mathrm{L}$, eGFR: $37 \mathrm{~mL} / \mathrm{min} / 1.73 \mathrm{~m}^{2}$ ). There was no sign of intravascular hemolysis on laboratory investigations and thrombotic microangiopathy was absent on allograft biopsies. Complement blockade was effectively achieved and residual eculizumab concentrations remained constantly $>400 \mu \mathrm{g} / \mathrm{mL}$.

Noëlle Cognard

noelle.cognard@chru-strasbourg.fr

1 Department of Nephrology and Transplantation, Strasbourg University Hospital, Strasbourg, France
On April 1st, 2020, the patient was hospitalized because of diarrhea, nausea, and flu-like symptoms that lasted for $24 \mathrm{~h}$, and was subsequently diagnosed with coronavirus disease 2019 (COVID-19). RT-PCR was conducted using a nasopharyngeal swab specimen and revealed that the patient was infected with severe acute respiratory syndrome coronavirus-2 (SARS-CoV-2) with a high viral load (7.57 $\log _{10}$ copies/reaction; Fig. 1). Chest computed tomography (CT) revealed bilateral ground-glass opacities with peripheral subpleural distribution, compatible with COVID-19 pneumonia with minimal parenchymal involvement $(<10 \%$; Fig. 2a). Eculizumab administration, which was initially scheduled for April 1st, was delayed and immunosuppression was reduced. MMF was discontinued on April 1st, whereas tacrolimus and steroids were maintained. On April 9th, the patient showed severe hypoxemia (required supplemental oxygen through a face mask: $15 \mathrm{~L} / \mathrm{min}$ ) requiring admission to the intensive care unit. A chest CT performed on April 11th revealed extension of pneumonia affecting approximately $50 \%$ of the lung parenchyma with alveolar condensation in the right lower lobes (Fig. 2b). Treatment was attempted with azithromycin (250 mg/day) and hydroxychloroquine (200 mg twice per day) but was rapidly discontinued because of electrocardiogram changes. Tacrolimus and prednisone were discontinued on April 8th. Dexamethasone (20 mg/day) was introduced on April 8th and subsequently tapered off (Fig. 1). Renal function remained stable (serum creatinine on April 4th: $128 \mu \mathrm{mol} / \mathrm{L}$ [reference range: 49-90 $\mathrm{mmol} / \mathrm{L}])$. There was evidence of a mild inflammatory syndrome (C-reactive protein: $30 \mathrm{mg} / \mathrm{L}$ [reference range: $<4 \mathrm{mg} / \mathrm{L}]$ ) but signs of thrombotic microangiopathy were absent (Fig. 3). Eculizumab was finally administered at a dose of $1200 \mathrm{mg}$ on April 9th (residual eculizumab plasma concentration: $220 \mu \mathrm{g} / \mathrm{mL}$ ). The patient showed a favorable clinical course with a progressive reduction in inflammatory markers paralleled by a decline in oxygen need. She was successfully discharged on April 23 and a further dose of eculizumab (900 mg) was administered. 


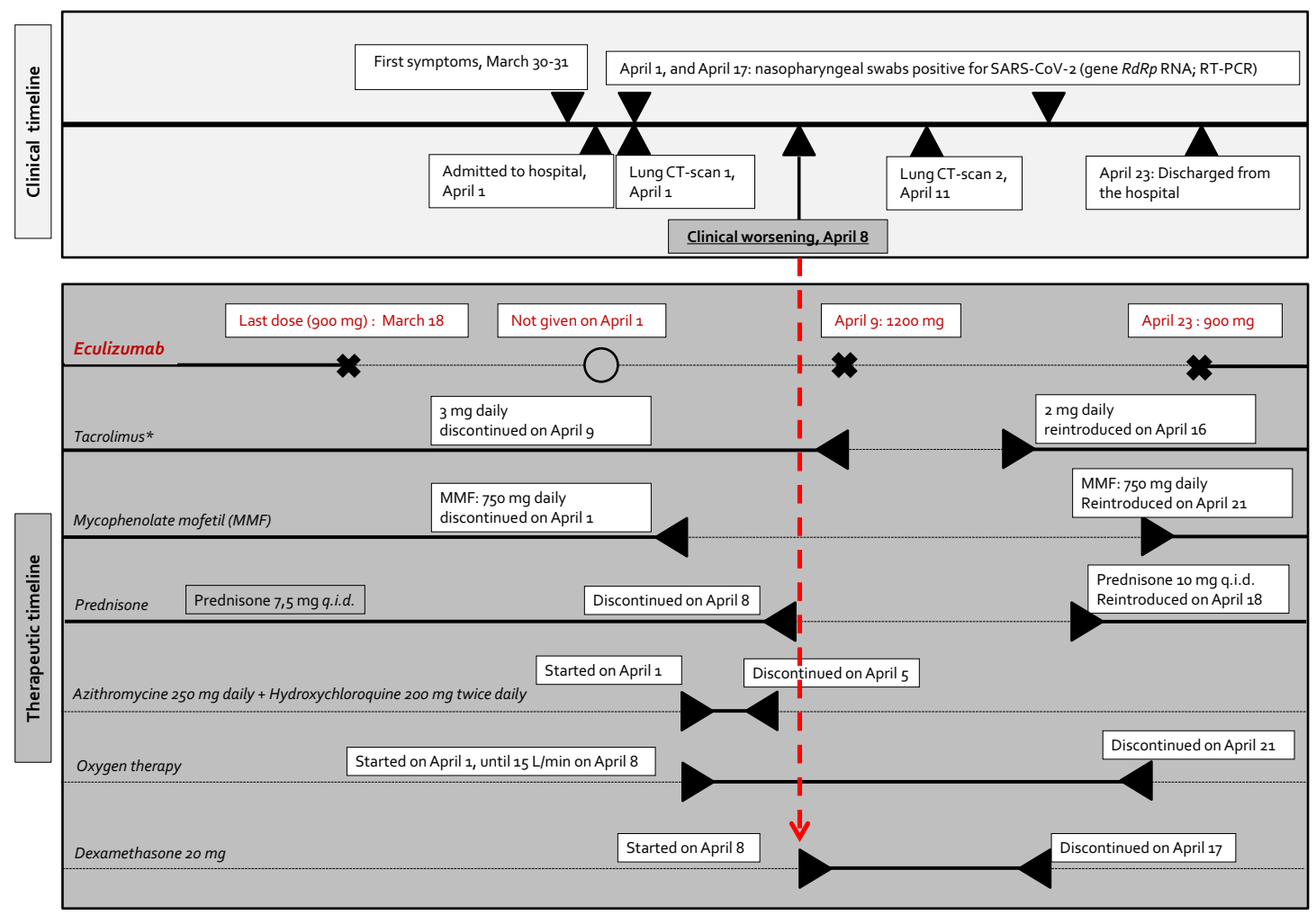

* Once daily prolonged release tacrolimus

Fig. 1 Timelineof the patient's clinical presentation and therapeutic management

Fig. 2 Lungcomputed tomography images acquired at the time of diagnosis (a) and at day 12 (b)after symptom onset. Panel a: bilateral ground-glass opacities in theperipheral subpleural areas. Panel b: extension of lesions to the lungparenchyma with evidence of alveolar condensation in the right lower pulmonarylobes

Fig. 3 Biochemical data on April 4th (3 daysafter hospitalization for coronavirus disease 2019)

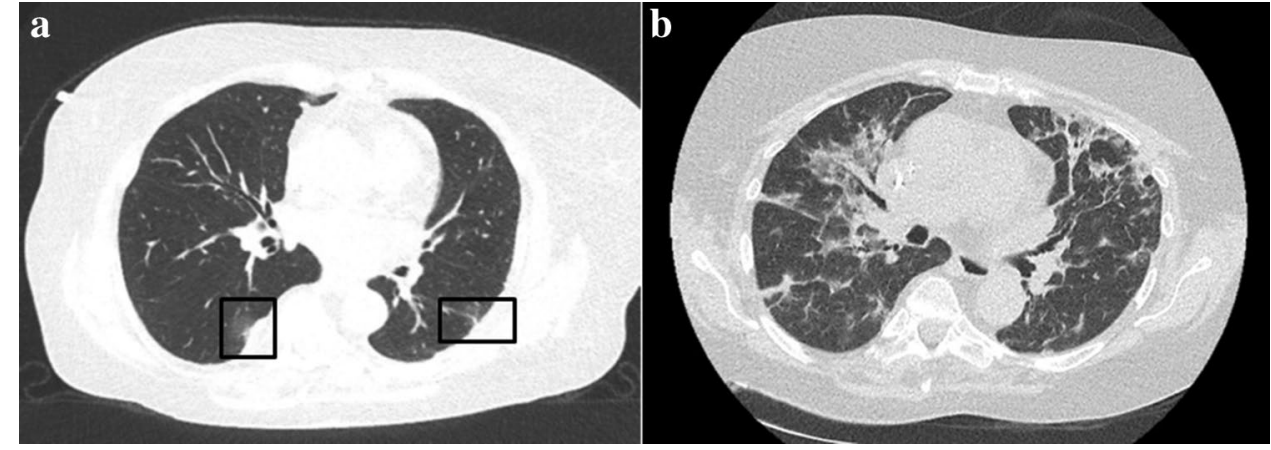

\begin{tabular}{|l|c|c|}
\hline & Biochemical Data & Reference range \\
\hline Serum créatinine & 128 & $49-90 \mu \mathrm{mol} / \mathrm{L}$ \\
\hline C-reactive protein & 30 & $<4 \mathrm{mg} / \mathrm{L}$ \\
\hline Hemoglobin & 11 & $12-16 \mathrm{~g} / \mathrm{dL}$ \\
\hline Platelets & 165 & $150-40010^{9} / \mathrm{L}$ \\
\hline LDH & 188 & $120-246 \mathrm{U} / \mathrm{L}$ \\
\hline Haptoglobin & 2.94 & $0.3-2 \mathrm{~g} / \mathrm{L}$ \\
\hline Schizocyte & absent & $/$ \\
\hline
\end{tabular}

SARS-CoV-2 may, at least in part, originate from dysregulation of innate and/or adaptive immune responses. Remarkably, excess stimulation of a host response during the course of COVID-19 can lead to an overwhelming 
release of proinflammatory cytokines-a phenomenon known as "cytokine storm"-leading to a dramatic deterioration of respiratory function and dismal outcomes [1]. Complement is involved in a large number of immune effector mechanisms during inflammatory processes, including the "cytokine storm."

Remarkably, complement activation plays a critical role in the pathogenesis of COVID-19, including endothelial cell damage [2]. In addition, in vitro studies have shown that SARS-CoV-2 spike proteins are capable of activating the complement alternative pathway [3]. Recent research revealed the presence of thrombotic microangiopathy and endothelial injury in patients infected with SARS-CoV-2, suggesting a functional role for complement activation in the pathogenesis of COVID-19 [4]. These findings provide a rationale for blocking complement activation in this clinical entity.

Eculizumab-a recombinant humanized monoclonal antibody that binds the $\mathrm{C} 5$ complement component and prevents its activation by cleavage- has been approved for treatment of paroxysmal nocturnal hemoglobinuria and aHUS. The antibody is used in the KT setting for preventing aHUS recurrences [5]. The pathogenesis of aHUS is related to an uncontrolled complement activation in the alternative pathway. Complement-mediated aHUS is clinically characterized by the presence of microangiopathic hemolytic anemia, thrombocytopenia, and acute renal failure. Acquired autoantibodies to complement regulators or genetic defects in complement-related factors have been repeatedly reported in patients with aHUS. Eculizumab is effective in preventing aHUS recurrences by inhibiting the production of the terminal complement component $\mathrm{C} 5 \mathrm{a}$ and the membrane attack complex C5b-9.

The case reported here describes a KT recipient diagnosed with COVID-19 who was treated with eculizumab to prevent recurrence of aHUS after her transplant. The first symptoms of COVID-19 occurred 12/13 days after the last eculizumab administration and, after much deliberation, the initially scheduled injection was postponed. Under the hypothesis that both complement activation and rapid eculizumab consumption (because of increased C5b-9 formation) should have occurred, a decision was made to administer eculizumab at a higher dose $(1200 \mathrm{mg})$. While drug-drug interactions have been shown to complicate COVID-19 treatment-and while there are contrasting reports on whether specific drugs worsen recovery-eculizumab did not hinder our patient's recovery from COVID19 [6]. Instead, the outcome was similar to that observed in most of our KT recipients with COVID-19 (i.e., nonsevere disease followed by recovery). Notably, Annane et al. have recently provided evidence that eculizumab may improve survival in patients with severe COVID-19 [7].
Nonetheless, the published literature on the clinical course of COVID-19 in patients treated with eculizumab remains limited. Trimarchi et al. have reported a case of a KT recipient with aHUS on chronic eculizumab therapy that developed severe COVID-19 despite eculizumab administration early during the course of the disease [8]. The outcome was favorable, and the patient recovered following dexamethasone and convalescent plasma administration. Diurno et al. have described four cases of severe disease treated with up to four weekly infusions of eculizumab (900 mg each) [9]. All these patients were immunocompetent, but one was aged 82 years. Concomitant treatments consisted of antiviral drugs, hydroxychloroquine, and ceftriaxone. Remarkably, treatment-related adverse effects were not reported. Outcomes were favorable with concomitant reduction in inflammatory biomarkers [9]. We cannot exclude that dexamethasone-which was used when clinical deterioration occurred-might have played a role in improving the patient clinical conditions through its anti-inflammatory effects.

Additionally, Ville et al. published a case of aHUS relapse in the context of COVID-19. These data show how COVID-19 can, on the one hand, be a trigger of aHUS relapse and, on the other hand, drive complement activation and increase C5b-9 formation (ultimately leading to eculizumab consumption) [10]. Thus, in accordance with previous literature, eculizumab did not hinder our patient's recovery from COVID-19—who did not have evident signs of recurrent thrombotic microangiopathy. Pulmonary and kidney injury in patients with COVID-19 may, at least partially, be mediated by complement activation. Terminal pathway inhibition by the anti-C5 antibody eculizumab can be included in the therapeutic armamentarium against COVID-19, and this treatment should not be discontinued unless strictly necessary due to adverse effects or alternative health complications.

Authors' contributions NC, GGV, PP, and IB: data acquisition. NC and SC: study conception and design. IB: analysis and interpretation of data. NC and SC: drafting of the manuscript. All authors have read and approved the final version of the paper for publication.

Funding This work received no specific funding.

Availability of data and materials The dataset used for this work can be requested from the corresponding author upon reasonable request.

\section{Declarations}

Ethics approval and consent to participate Collection of data was accepted by the local ethics committee (DC-2013-1990). The patient signed an informed consent for data collection. 
Consent for publication Written informed consent was obtained from the patient to publish this case report and any accompanying images. Patient data has been previously published.

Conflict of interest NC, GGV, PP, IB, and SC declare no competing interests.

\section{References}

1. Ye Q, Wang Be, Mao J (2020) The pathogenesis and treatment of the 'Cytokine Storm' in COVID-19. J Infect 80:607-613

2. Noris M, Benigni A, Remuzzi G (2020) The case of complement activation in COVID-19 multiorgan impact. Kidney Int 98:314-322

3. Yu J, Yuan X, Chen $\mathrm{H}$ et al (2020) Direct activation of the alternative complement pathway by SARS-CoV-2 spike proteins is blocked by factor D inhibition. Blood 136:2080-2089

4. Campbell CM, Kahwash R (2020) Will complement inhibition be the new target in treating COVID-19 related systemic thrombosis? Circulation. doi:https://doi.org/10.1161/CIRCULATIONAHA. 120.047419

5. Legendre CM, Licht C, Muus P et al (2013) Terminal complement inhibitor eculizumab in atypical hemolytic-uremic syndrome. $\mathrm{N}$ Engl J Med 368:2169-2181
6. Awortwe C, Cascorbi I (2020 Nov) Meta-analysis on outcomeworsening comorbidities of COVID-19 and related potential drugdrug interactions. Pharmacol Res 161:105250

7. Annane D, Heming N, Grimaldi-Bensouda L et al (2020) Eculizumab as an emergency treatment for adult patients with severe COVID-19 in the intensive care unit: a proof-of-concept study. EClinicalMedicine 28:100590

8. Trimarchi H, Gianserra R, Lampo M et al (2020) Eculizumab, SARS-CoV-2 and atypical hemolytic uremic syndrome. Clin Kidney J 13(5):739-741

9. Diurno F, Numis FG, Porta G et al (2020) Eculizumab treatment in patients with COVID-19: preliminary results from real life ASL Napoli 2 Nord experience. Eur Rev Med Pharmacol Sci 24(7):4040-4047

10. Ville S, Le Bot S, Chapelet-Debout A et al (2021 Jan) Atypical HUS relapse triggered by COVID-19. Kidney Int 99(1):267-268

Publisher's note Springer Nature remains neutral with regard to jurisdictional claims in published maps and institutional affiliations. 